\title{
DICER1-Mutated Botryoid Fibroepithelial Polyp of the Parotid Duct: Report of the First Case
}

\author{
Ramona Erber ${ }^{1,2} \cdot$ Raimund Preidl $^{2,3} \cdot$ Robert Stoehr $^{1,2} \cdot$ Florian Haller $^{1,2} \cdot$ Arndt Hartmann $^{1,2} \cdot$ Marco Kesting $^{2,3}$. \\ Abbas Agaimy ${ }^{1,2}$ (D)
}

Received: 1 June 2021 / Accepted: 7 July 2021 / Published online: 19 July 2021

(c) The Author(s) 2021

\begin{abstract}
DICER1, a member of the ribonuclease III family, is involved in the biogenesis of microRNAs and, hence, it influences gene expression regulation. DICERI germline (associated with the inherited DICERI syndrome) or somatic mutations have been linked to tumorigenesis in histogenetically diverse benign and malignant neoplasms in different organs including pleuropulmonary blastoma, cystic nephroma, embryonal rhabdomyosarcoma, nasal chondromesenchymal hamartoma, poorly differentiated thyroid carcinoma, thyroblastoma, intracranial sarcoma and gonadal Sertoli-Leydig cell tumors in addition to others. Moreover, rare botryoid (giant) fibroepithelial polyps may harbor this mutation. Herein, we describe the first reported case of a DICER1-mutated botryoid fibroepithelial polyp occurring within the parotid duct of a 65 -year-old female who has no other features or family history of the DICERl syndrome. Based on its distinctive morphology, we tested this lesion specifically for DICER1 mutations and confirmed the presence of a pathogenic DICERl variant with a low allele frequency, consistent with a somatic mutation.
\end{abstract}

Keywords DICERI - Mutations · Salivary gland · Parotid duct · Fibroepithelial polyp · Botryoid polyp

\section{Introduction}

DICER 1, located on the long arm of chromosome 14 (14q32.13, OMIM\# 606241), encodes for Dicer protein which belongs to the ribonuclease III family and influences the activity of other genes by regulation of microRNA (miRNA) biogenesis [1]. DICERI mutations are involved in deregulation of miRNA and, hence, influence tumorigenesis [2]. During the last decade, DICERI mutations have been increasingly recognized to be associated with diverse

Ramona Erber and Raimund Preidl have contributed equally.

Abbas Agaimy

abbas.agaimy@uk-erlangen.de

1 Institute of Pathology, Friedrich-Alexander University Erlangen-Nürnberg (FAU), University Hospital Erlangen (UKER), Krankenhausstrasse 8-10, 91054 Erlangen, Germany

2 Comprehensive Cancer Center Erlangen-EMN (CCC ER-EMN), Erlangen, Germany

3 Department of Oral and Maxillofacial Surgery, FriedrichAlexander University Erlangen-Nürnberg (FAU), University Hospital Erlangen (UKER), Erlangen, Germany benign and malignant neoplastic entities with distinctive morphological features that represent clues to an underlying DICER 1 mutation. While the majority of DICER1 mutations represent germline variants in the setting of the inherited DICER 1 syndrome, sporadic diseases with DICER1 mutations have been recently recognized, albeit rare. Individuals affected by germline DICERI mutations tend to develop multinodular goiter [2], and have an increased risk of developing a variety of tumors, including pleuropulmonary blastoma [2], Wilms tumor (with low frequency) [3], cystic nephroma, embryonal rhabdomyosarcoma, nasal chondromesenchymal hamartoma [4], gynandroblastoma [5], esophageal polyps [6], medulloblastoma [7], and Sertoli-Leydig cell tumors of the ovary $[2,8]$. To date, $>1136$ DICER1 variants (both germline and somatic) comprising protein truncating, in-frame deletions, and missense mutations have been described in $>808$ individuals $[2,6,8,9]$. Recently, we have described a botryoid fibroepithelial polyp of the urinary bladder carrying a DICER1 mutation [10]. We herein describe the first reported case of a DICERI-mutated botryoid fibroepithelial polyp of the parotid duct. 


\section{Clinical History}

A 65- year- old woman presented with a progressively growing painless mass in her left buccal mucosa for 8 weeks. The mass was mobile and palpable in the region of the parotid duct next to the parotid papilla. There was no purulence or evidence of total parotid duct obstruction. The patient's medical history was negative for neoplasms or other significant co-morbidities. Her family history revealed that the patient's mother died of pancreatic cancer at age 56. Her father and three sisters had no cancer history. Preoperative MRI revealed a solid mass meassuring $1.3 \times 1.0 \times 0.9 \mathrm{~cm}$ located adjacent to the left masseter muscle with partial compression of the parotid duct. The mass was only partially contrast-enhancing and was hypointense in $\mathrm{T} 1$ sequence and hyperintense in T2 sequence (Fig. 1A, B).

Intraoperatively, the mass was well-demarkated from the surrounding soft tissue without infiltration. It was resected together with the adjacent parotid duct and the papilla with reinsertion of the parotid duct posterior to its original location (Fig. 1C-E). A splinting was fixed within the duct to maintain patency. The postoperative course was unremarkable, and the patient was released on the 3rd postoperative day.

\section{Material and Methods}

The surgical specimen was fixed in formalin and embedded routinely for histopathological evaluation. Hematoxylin and eosin (H\&E) stained slides were prepared for histopathological examination and immunohistochemical staining was performed on $1 \mu \mathrm{m}$ thick sections using an automated platform (Benchmark Ultra, Ventana Medical Systems Inc., Tucson, Arizona, U.S.A.) and the following antibodies: CD34 (clone QBEND-10; 1:50, Immunotech), smooth muscle actin (clone 1A4, 1:400, Dako), desmin (clone D33, 1:50, Dako), MyoD1 (clone 5.8A, 1:50, Dako), myogenin (clone F5D, 1:50, Dako), S100 (clone 4C4.9, 1:3000, Zytomed), MDM2 (clone IF2, 1:50, Calbiochem), CDK4 (clone DCS-156, 1:100, Zytomed), Retinoblastoma-1 (clone G3-245, 1:100, BD Pharmingen), CD10 (clone 56C6, 1:20, Zytomed), STAT6 (clone S-20, 1:1000, Santa Cruz) and SATB2 (clone EPNCIR130A, 1:200, abcam).

\section{Molecular Testing}

After careful manual microdissection, DNA was extracted from FFPE tumor tissue using the Maxwell ${ }^{\odot} 16$ system (Promega, Madison, Wisconsin, USA) according to manufacturer's instructions. DICERI sequence analysis was performed using the QIAseq Targeted Human Comprehensive Cancer Panel encompasing 160 cancer-related genes according to manufacturer's instructions. Bioinformatic evaluation of the sequencing data, including variant calling and annotation, was done with the CLC Genomics Workbench (QIAGEN, Redwood City, CA, USA). Low quality variants with a score under 200 were filtered out, as well as variants in non-protein-coding regions, synonymous variants, and those present in GnomAD with an allele frequency of over $2 \%$. The remaining variants were assessed for pathogenicity according to ACMG/AMP criteria. The DICERI variants were classified as described previously [11].

\section{Results}

\section{Pathological Findings}

Grossly, the parotid duct showed cystic dilatation measuring $1.5 \times 1.0 \times 0.9 \mathrm{~cm}$. It was filled with a lobulated soft mass with smooth surface (Fig. 1F). Histologically, the dilated salivary duct was lined by a single layer of columnar epithelium with interspersed goblet cells and non-mucinous columnar cells interrupted by foci of ciliated epithelium without atypia. Within the lumen, there was a large fibroepithelial lesion reminiscent of a phylloides tumor composed of variably edematous or fibrous leaflets covered by similar epithelium as the original duct (Fig. 2A, B). Irregularly distributed sebaceous elements, frequeuntly merging with the respiratory epithelium, were seen beneath the epithelium covering the leaflets (Fig. 2C, D). The epithelium covering the papillary leaflets was similar to and continuous with the luminal epithelium lining the duct but displayed hyperplastic features with increased number of goblet cells and continuous basal cell layer (Fig. 3A-D). Focal aggregates of serous acini were seen within the subepithelial stroma (Fig. 3D), as well as sebaceous elements (Fig. 2C, D). The stroma was moderately cellular, composed of fibroblast-like spindle cells with variable ectatic vessels (Fig. 4A, B), sparse mononuclear inflammatory infiltrates (Figs. 2D \& 3B), and focal multinucleated stromal giant cells (Fig. 4C). Foci of micronodular fibromyxoid stromal changes were seen (Fig. 2C). Immunohistochemistry showed a variable expression of CD34 in the spindle cells (Fig. 4D) and multinucleated giant cells (Fig. 4E). Desmin was positive in a few spindled stromal cells with strong expression in the multinucleated giant cells (Fig. 4F). Other markers (STAT6, MyoD1, myogenin, SATB2, S100, MDM2, CDK4, and smooth muscle actin) were negative. Retinoblastoma-1 protein displayed retained expression. 

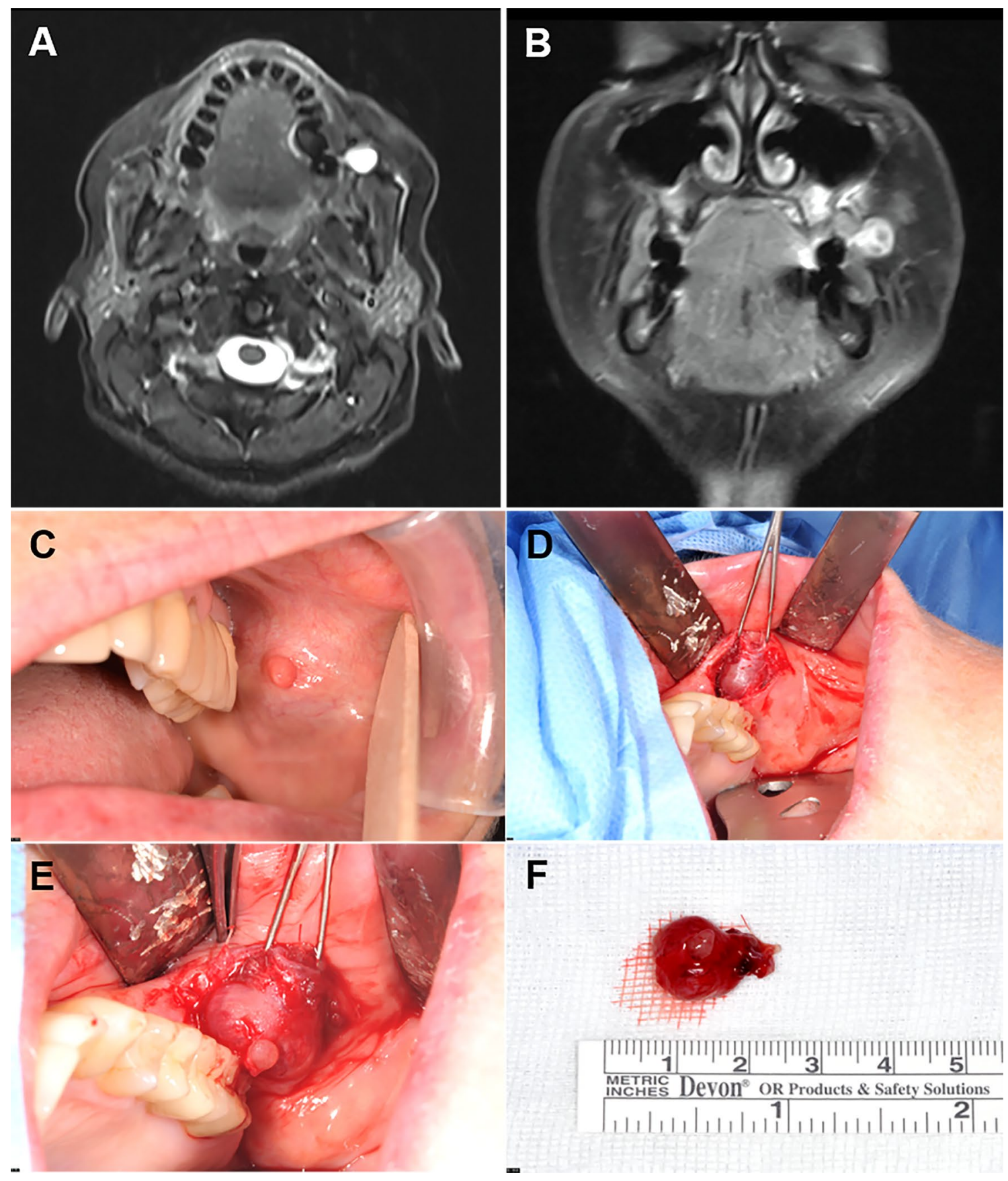

\section{$\mathbf{F}$}

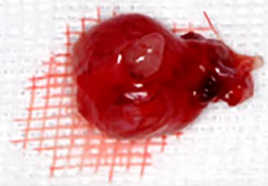

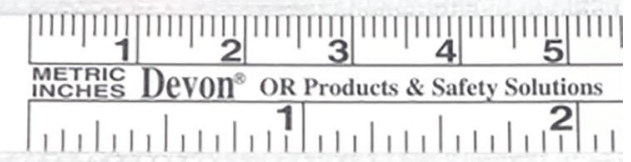

Fig. 1 Preoperative MRI showed a mass in the left buccal region with hyperintense signaling in T2 sequence (A) and partially contrastenhancing and hypointense signaling in T1 sequence (B). Clinically, the mass was closely associated with the papilla of the parotid duct (C). Intraoperatively, the duct was found to run through the lesion

\section{Molecular Findings}

The molecular analysis revealed a DICER1 mutation (p. [Pro1645fs]; ENST00000343455: c.[4933_4935delCCAinsAG]) with an allele frequency of $7.5 \%$. Sequencing depth at the mutation site was $254 \times$. With

which required a cannulation of the residual duct after total resection with a reinsertion of the duct posterior to its original location $(\mathbf{D}, \mathbf{E})$. F Gross specimen of the resected mass showing a well circumscribed glistening surface

a tumor cell content of $>40 \%$ within the microdissected and analyzed tissue, the variant appears to be a somatic, heterozygous event. The detected mutation is located within exon 23 of DICERI and the affected amino acid is positioned within the functional domain between the RNase IIIa and RNase IIIb domains of DICERI [8]. The resulting frameshift 


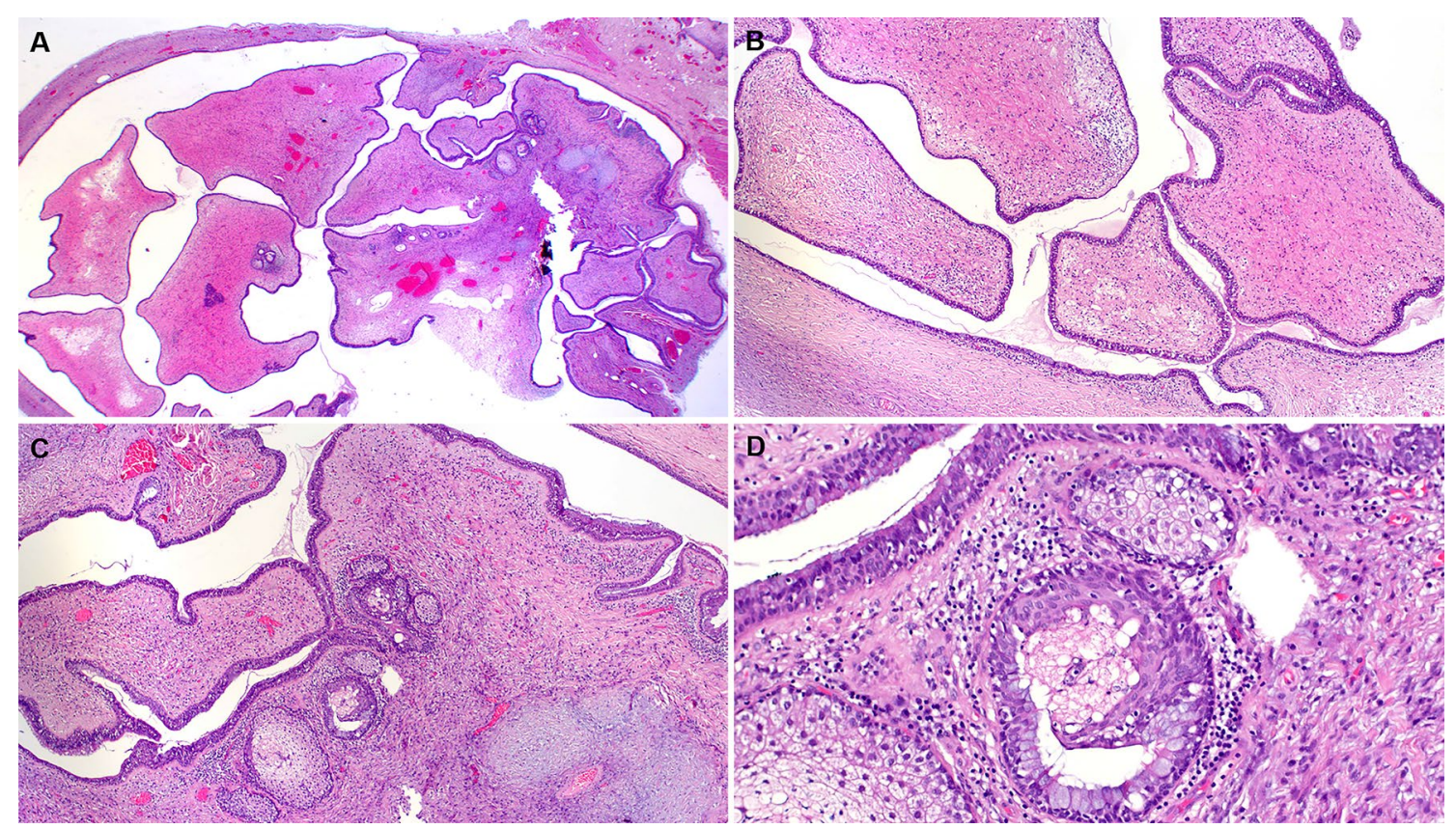

Fig. 2 A Overview of the histopathological specimen showing phylloides-like fibroepithelial lesion within dilated duct, covered by squamous oral mucosa (upper right field). B the lesion was composed of plump edematous to fibrous leaflet-like projections within the cystic cavity, covered by columnar respiratory-type epithelium continuous with the mucosal lining of the cystic duct. C Sebaceous elements and focal myxoid change are seen within the stroma. D The sebaceous glands merge with the lining epithelium (mid-lower field)

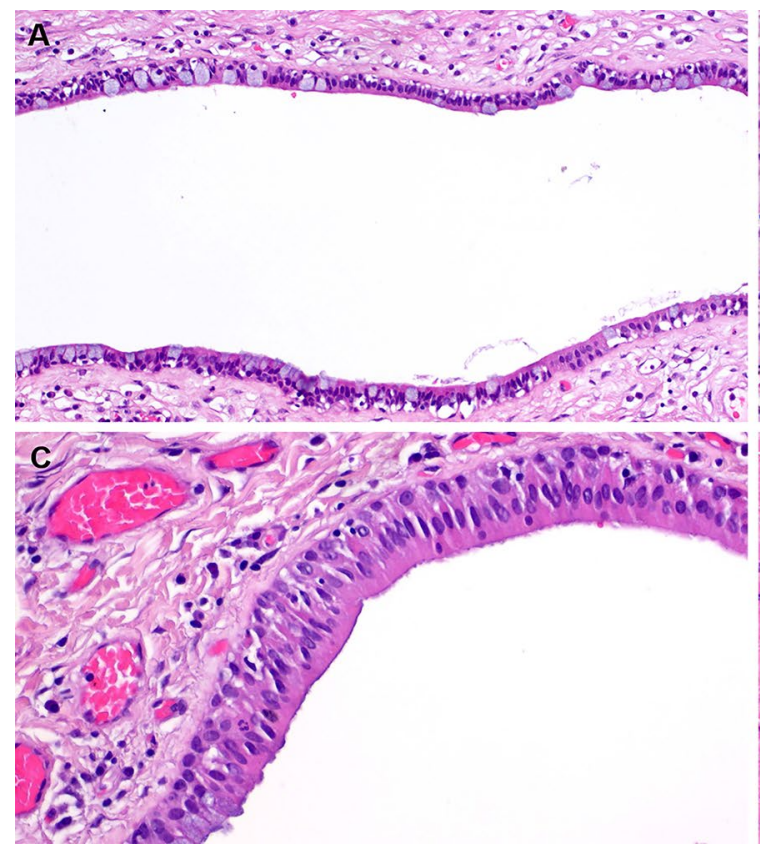

Fig. 3 The epithelium lining the cystic duct (upper field) and the epithelial component covering the leaflets (lower field) are more or less similar (A), but the latter shows variable hyperplastic changes (B, right). C The mucus cell-containing epithelium (lower left) merges

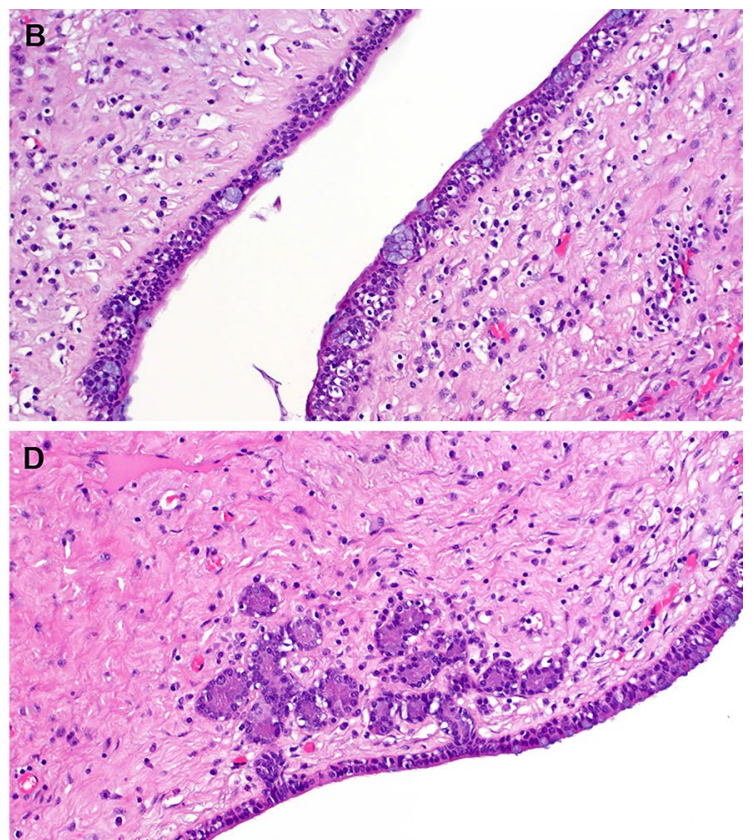

with eosinophilic columnar ciliated cells lacking mucous elements (C). D salivary-type serous acini closely associated with the epithelium covering the leaflets 

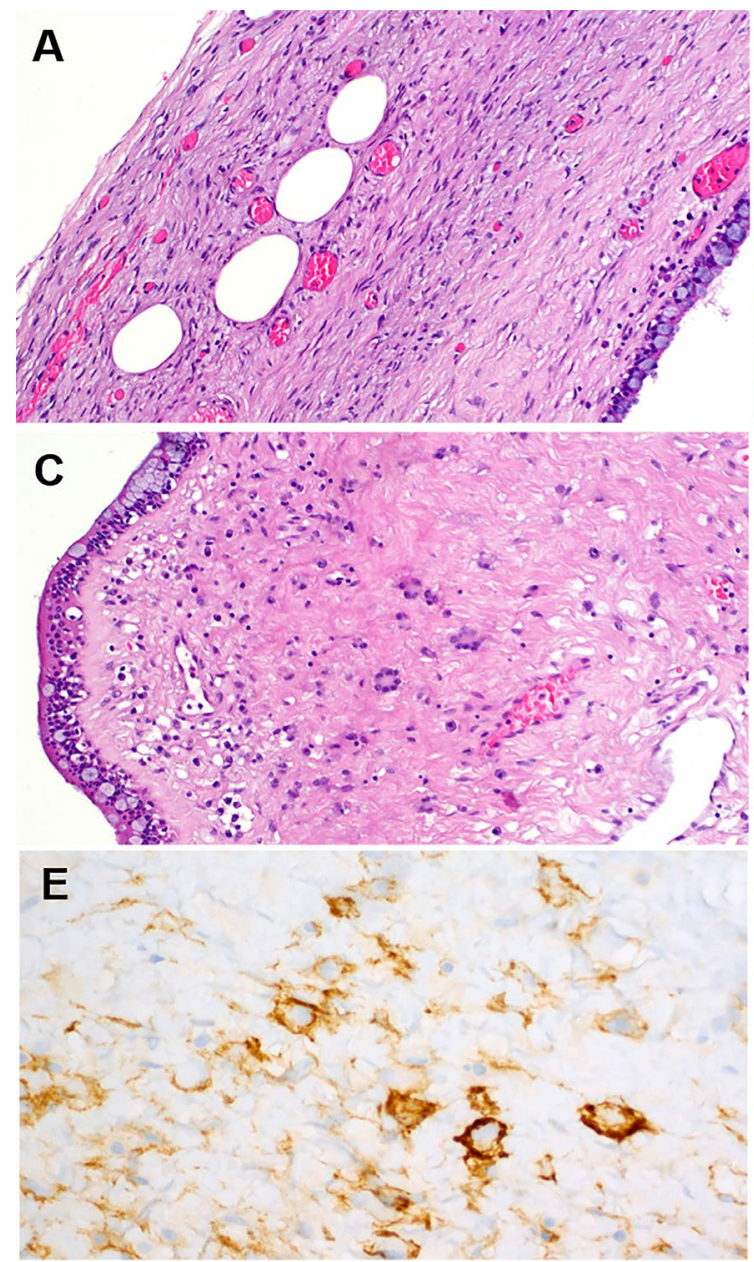

Fig. 4 The stromal component was mainly composed of fibroblastic spindle cells entrapping single fat cells at the periphery of the lesion (A). B higher magnification of the spindle cells, note ectatic vessels. C scattered multinucleated stromal giant cells were seen

affects the functionally crucial RNase IIIb domain suggesting a loss of function effect of the detected variant.

\section{Discussion}

In this case report, we described a "giant" botryoid fibroepithelial polyp of the parotid duct with DICERI mutation in an adult female patient. To the best of our knowledge, this is the first documentation of this finding in the salivary glands. Fibroepithelial polyps are polypoid overgrowths of epithelial elements and stromal tissue usually protruding into a hollow organ, a cavity, or overlying a body surface. The epithelial component usually recapitulates the normal epithelium of the organ of origin but may show hyperplastic, metaplastic, or hamartoma-like features. Other mesenchymal elements such as mature skeletal muscle and (rarely) cartilage may be present.
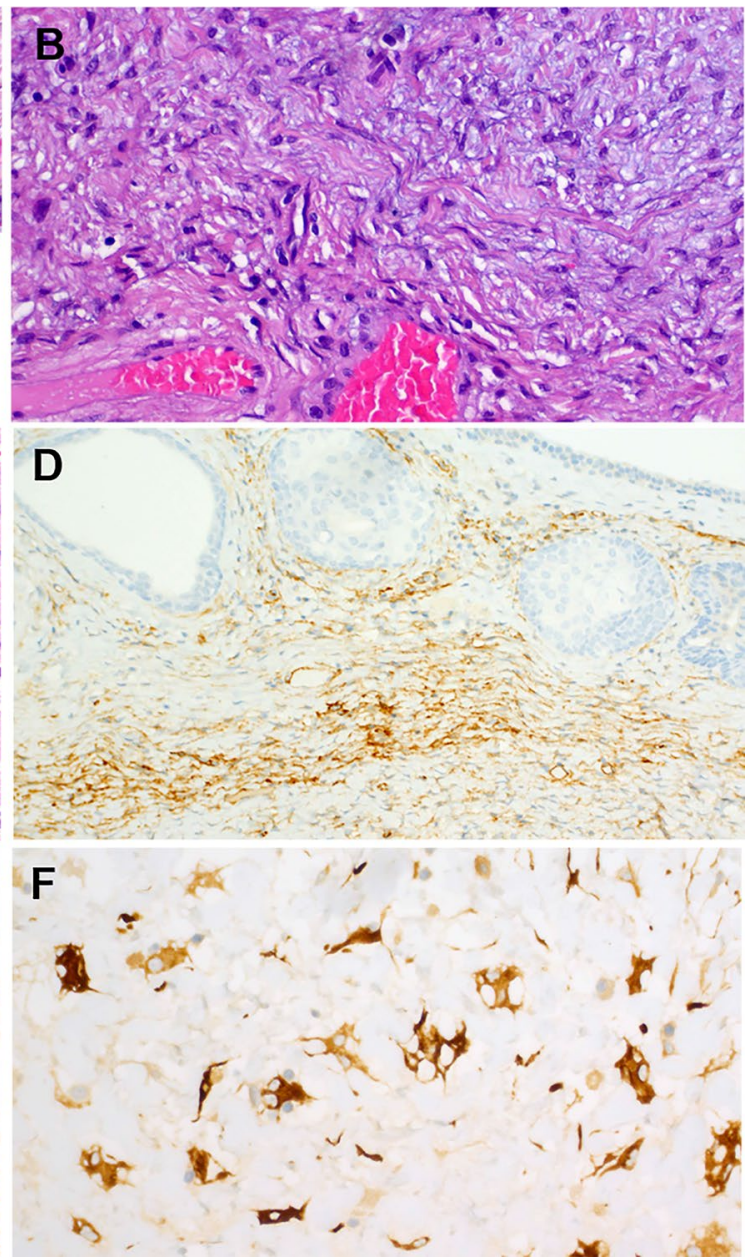

focally. Immunohistochemistry showed experssion of CD34 in the spindle cells (D) and the multinucleated giant cells (E). Desmin was expressed strongly in the giant cells (highlighting prominent dendritic cytoplasmic processes) and variably in the spindle cells $(\mathbf{F})$

Simple fibroepithelial polyps however are not uncommon. They mainly occur in the skin, where they are also designated as acrochordons or skin tags [12-16]. They also occur in the genitourinary tract and in the oral cavity (predominantly in the buccal area [16-18]. Rare sites include the orbita [12], larynx [19, 20], tonsil [21, 22], bronchi [23], nasal septum [24], inferior nasal turbinate [25], middle ear [26], and the external auditory canal [27]. Occasinally, these polyps may contain a variable amount of sebaceous glands [28]-30]. Fibroepithelial polyps behave mostly in a benign fashion; recurrences after complete excision are rare [31-34].

Large (up to $18.5 \mathrm{~cm}$ ) fibroepithelial polyps growing into a cauliflower-like (botryoid) pattern have been rarely documented in several anatomical sites [35]. They have been reported in the urinary bladder [10], anus [36] and the nipple [37]. Uncommon sites include the ureter, glans penis, vagina, vulva, perineum and skin. Due to occasional 
persence of atypical or bizzare-looking stromal cells, multinucleated cells, and myxoid stromal changes, these polyps have historically been termed pseudosarcoma botryoides or pseudosarcomatous polyps [31-35, 38-43]. Grossly and histopathologically, botryoid fibroepithelial polyps may be indistinguishable from botryoid embryonal rhabdomyosarcoma. Hence, their differential diagnosis includes, amongst other lesions, embryonal rhabdomyosarcoma [44], as well as other entities, based on the anatomic location of the lesion $[35,45]$.

The etiology of fibroepithelial polyps is unknown. A reactive etiology (due to localized trauma or tissue irritation) and a congenital origin have been proposed as potential explanations [46]. Notably, data on genetic findings in botryoid or giant fibroepithelial polyps is scarce. We recently have described one giant botryoid fibroepithelial polyp of the urinary bladder, which showed two non-synonymous DICERI pathogenic variants [10]. Reviewing the English literature thoroughly, we were not able to find any more reports on this topic and, to our knowledge, similar botryoid polyps have not been reported in the salivary glands.

Based on its distinctive morphology, we examined the current lesion specifically for DICERI mutations. With increasing awareness of their distinctive morphology and the use of modern genetic tools, DICERI-associated lesions and neoplasms (both benign and malignant) are becoming increasingly recognized in surgical pathology. These tumors are generally characterized and unified by a biphasic epithelial and mesenchymal growth with the epithelial component essentially reflecting the original epithelium of the organ of origin or its embryonal counterpart, while the stromal component varies greatly based on the tissue of origin, the biology of the entity (whether benign or malignant) and the presence or absence of heterologous mesenchymal tissue derivatives. Accordingly, the patterns seen in many of the DICER I-related neoplasms are most likely fibroepithelial or phylloides-like (in benign lesions) or adenosarcomaor blastoma-like (in malignancies), with some neoplasms showing an admixture of diverse primitive tissue elements resulting in a teratoid appearance. In the head and neck, chondromesenchymal sinonasal hamartoma (mostly hereditary [4]) and thyroblastoma (likely sporadic [47]) represent the most well characterized DICERI-related manifestations. Among dental abnormalities and phenotypes associated with the DICER1 syndrome are bulbous crown, periodontitis, and taurodontism [48].

DICER 1 mutations predominantly represent germline variants but may also occur as sporadic (somatic) events. Individuals with the inherited DICER1 syndrome are at increased risk to develop various tumors including pleuropulmonary blastoma [2], Wilms tumor [3], embryonal rhabdomyosarcoma, Sertoli-Leydig cell tumors of the ovary [2], medulloblastoma, cystic nephroma of the kidney, poorly differentiated thyroid carcinoma, intracranial sarcoma, and, more recently identified, pleuropulmonary blastoma-like abdominopelvic sarcoma [49]. Accordingly, the patient's medical and family history should be checked thoroughly for DICERI-suspicious neoplasms. In our case, the DICERI pathogenic variant was associated with a low allele frequency, which argued against a germline mutation, consistent with the fact that in the presented case, the patient had no medical or family history of DICERI-related lesions.

In summary, we herein describe the first reported case of a botryoid fibroepithelial polyp occurring within the parotid gland duct and carrying a, likely somatic, pathogenic DICERI variant. Recognition of this rare presentation is necessary for clinical and genetic work-up of the affected patients. This case nicely highlights the value of standard morphology in predicting the genotype in specific entities which would otherwise have gone unrecognized as being associated with a specific genetic background. Although the current case is likely sporadic, it is important to recognize these rare lesions. Identification of more cases in the future would allow to assess their possible association with the DICER1 syndrome more reliably.

Acknowledgements Special thanks to Patricia Segschneider and Karola Rudelt for technical support.

Author Contributions RE, RP, AA: Conception and design of the work, acquisition, analysis and interpretation of data, drafting the MS and revising it critically for important intellectual content and scientific integrity. RE, RP, RS, FH, AH, MK, AA: Acquisition, analysis and interpretation of data, reading and revising the MS critically for important intellectual content and scientific integrity. All authors have read and approved the final manuscript.

Funding Open Access funding enabled and organized by Projekt DEAL. No external funding was obtained for this study.

\section{Declarations}

Conflict of interest All authors declare that they have no conflict of interest as it relates to this research project.

Ethical Approval Samples were used in accordance with ethical guidelines for the use of retrospective tissue samples provided by the local ethics committee of the Friedrich-Alexander University ErlangenNuremberg (Ethics committee statements 24.01.2005 and 18.01.2012). All procedures performed in this retrospective data analysis involving human participants were in accordance with the ethical standards of our institutional review board.

Informed Consent Informed consent has been obtained.

Open Access This article is licensed under a Creative Commons Attribution 4.0 International License, which permits use, sharing, adaptation, distribution and reproduction in any medium or format, as long as you give appropriate credit to the original author(s) and the source, provide a link to the Creative Commons licence, and indicate if changes were made. The images or other third party material in this article are 
included in the article's Creative Commons licence, unless indicated otherwise in a credit line to the material. If material is not included in the article's Creative Commons licence and your intended use is not permitted by statutory regulation or exceeds the permitted use, you will need to obtain permission directly from the copyright holder. To view a copy of this licence, visit http://creativecommons.org/licenses/by/4.0/.

\section{References}

1. Murchison EP, Hannon GJ. miRNAs on the move: miRNA biogenesis and the RNAi machinery. Curr Opin Cell Biol. 2004;16(3):223-9.

2. Rio Frio T, et al. DICER1 mutations in familial multinodular goiter with and without ovarian Sertoli-Leydig cell tumors. JAMA. 2011;305(1):68-77.

3. Palculict TB, et al. Identification of germline DICER1 mutations and loss of heterozygosity in familial Wilms tumour. J Med Genet. 2016;53(6):385-8.

4. Stewart DR, et al. Nasal chondromesenchymal hamartomas arise secondary to germline and somatic mutations of DICER1 in the pleuropulmonary blastoma tumor predisposition disorder. Hum Genet. 2014;133(11):1443-50.

5. Schultz KAP, et al. DICER1-related Sertoli-Leydig cell tumor and gynandroblastoma: clinical and genetic findings from the International Ovarian and Testicular Stromal Tumor Registry. Gynecol Oncol. 2017;147(3):521-7.

6. Foulkes WD, et al. Extending the phenotypes associated with DICER1 mutations. Hum Mutat. 2011;32(12):1381-4.

7. Slade I, et al. DICER1 syndrome: clarifying the diagnosis, clinical features and management implications of a pleiotropic tumour predisposition syndrome. J Med Genet. 2011;48(4):273-8.

8. de Kock L, Wu MK, Foulkes WD. Ten years of DICER1 mutations: provenance, distribution, and associated phenotypes. Hum Mutat. 2019;40(11):1939-53.

9. Verrier F, et al. Mutiple DICER1-related lesions associated with a germline deep intronic mutation. Pediatr Blood Cancer. 2018;65(6):e27005.

10. Eckstein M, et al. DICER1 mutation-positive giant botryoid fibroepithelial polyp of the urinary bladder mimicking embryonal rhabdomyosarcoma. Hum Pathol. 2019;84:1-7.

11. Richards S, et al. Standards and guidelines for the interpretation of sequence variants: a joint consensus recommendation of the American college of medical genetics and genomics and the association for molecular pathology. Genet Med. 2015;17(5):405-24.

12. Cho WK, et al. A case of congenital orbital fibroepithelial polyp. Ophthalmic Plast Reconstr Surg. 2013;29(4):e107-8.

13. Uğras $\mathrm{S}$, et al. Fibroepithelial polyp of the ureter associated with an adjacent ureteral calculus. Int Urol Nephrol. 1997;29(5):543-9.

14. Lipoff JB, Masood S, Chatterjee K. Acrochordon in statpearls. Treasure Island: StatPearls Publishing LLC; 2020.

15. Wali V, Wali VV. Assessment of various biochemical parameters and BMI in patients with skin tags. J Clin Diagn Res. 2016;10(1):BC09.

16. Şencan A, et al. A rare cause of glans penis masses in childhood: fibroepithelial polyp. J Indian Assoc Pediatr Surg. 2015;20(1):42-4.

17. Cooke BE. The fibrous epulis \& the fibro epithelial polyp: their histogenesis \& natural history. Br Dent J. 1952;93:305-9.

18. Mishra A, Pandey RK. Fibro-epithelial polyps in children: a report of two cases with a literature review. Intractable Rare Dis Res. 2016;5(2):129-32.

19. Jabbour J, et al. Glottic obstruction from fibroepithelial polyp. Am J Case Rep. 2019;20:219-23.

20. Farzal Z, Ulualp SO, Rakheja D. Fibroepithelial polyp of the epiglottis. Am J Case Rep. 2014;15:340-2.
21. Telugu RB, Ashish G. Fibroepithelial polyp of the tonsil: report of a rare case. J Clin Diagn Res. 2015;9(12):ED17-8.

22. Farboud A, et al. Fibroepithelial polyp of the tonsil: case report of a rare, benign tonsillar lesion. J Laryngol Otol. 2010;124(1):111-2.

23. Amin PB, Baciewicz F. Benign fibroepithelial polyp arising in the bronchus: a case report and review of the literature. Arch Surg. 2009;144(11):1081-3.

24. Stoiljkov M, Perić A. Fibroepithelial polyp originating from the nasal septum. Turk Arch Otorhinolaryngol. 2019;57(4):206-8.

25. Peric A, Matkovic-Jozin S, Vukomanovic-Durdevic B. Fibroepithelial polyp arising from the inferior nasal turbinate. J Postgrad Med. 2009;55(4):288-9.

26. Schuster D, et al. A case of sensorineural hearing loss involving a fibroepithelial polyp of the middle ear. Am J Otolaryngol. 2015;36(3):475-8

27. Tanaka N, Matsunobu T, Shiotani A. Fibroepithelial polyp of the external auditory canal: a case report and a literature review. Case Rep Otolaryngol. 2013;2013:818197.

28. Takeda Y, et al. Sebaceous gland hyperplasia in an intraoral fibrous polyp. Pathol Int. 2004;54(11):877-9.

29. Rajeesh Mohammed PK, et al. Fibroepithelial polyp with sebaceous hyperplasia: a case report. Indian J Med Paediatr Oncol. 2017;38(3):404-6.

30. Lloyd S, Lloyd J, Dhillon R. Chondroid metaplasia in a fibroepithelial polyp of the tongue. J Laryngol Otol. 2001;115(8):681-2.

31. Rexhepi M, et al. Giant fibroepithelial polyp of vulva: a case report and review of literature. Prilozi. 2018;39(2-3):127.

32. Khalil AM, et al. Vulvar fibroepithelial polyp with myxoid stroma: an unusual presentation. Gynecol Oncol. 1994;53(1):125-7.

33 Gupta M, et al. Giant fibroepithelial polyp of the ureter. BMJ Case Rep. 2017;2017:bcr2016218999.

34. Orosz Z, et al. Recurrent giant fibroepithelial stromal polyp of the vulva associated with congenital lymphedema. Gynecol Oncol. 2005;98(1):168-71.

35. Madueke-Laveaux OS, Gogoi R, Stoner G. Giant fibroepithelial stromal polyp of the vulva: largest case reported. Ann Surg Innov Res. 2013;7:8-8.

36. Galanis I, et al. Obstructive ileus due to a giant fibroepithelial polyp of the anus. World J Gastroenterol. 2009;15(29):3687-90.

37. Shaaban AM, Turton EPL, Merchant W. An unusual case of a large fibroepithelial stromal polyp presenting as a nipple mass. BMC Res Notes. 2013;6:345-55.

38 Ostör AG, Fortune DW, Riley CB. Fibroepithelial polyps with atypical stromal cells (Pseudosarcoma botryoides) of vulva and vagina. A report of cases. Int J Gynecol Pathol. 1988;7(4):351-60.

39. Pharaon M, Warrick J, Lynch MC. Fibroepithelial stromal polyp of the vulva: case report and review of potential histologic mimickers. Int J Gynecol Pathol. 2018;37(4):e1-5.

40. Colak E, et al. Giant fibroepithelial polyp of the perineum: giant fibroepithelial polyp. Int J Surg Case Rep. 2015;17:126-7.

41. Cathro HP, Patterson JW, Wick MR. Cutaneous pseudosarcomatous polyp: a recently described lesion. Ann Diagn Pathol. 2008;12(6):440-4.

42 Yan H, et al. Giant fibroepithelial polyp of the glans penis not associated with condom-catheter use: a case report and literature review. Can Urol Assoc J. 2013;7(9-10):E621-4.

43. Kumar A, et al. Giant fibro epithelial polyp in a young girl: a rare case report. Int J Surg Case Rep. 2017;38:83-5.

44. Silva Cunha JL, et al. Embryonal Rhabdomyosarcoma (botryoid subtype) affecting the buccal mucosa. Head Neck Pathol. 2019;13(4):671-6

45. Mercer SE, et al. Verrucous carcinoma masquerading as a giant fibroepithelial polyp. Int J Surg Pathol. 2010;18(5):347-51.

46. Vatansever M, et al. Atypical presentation of fibroepithelial polyp: a report of two cases. Arq Bras Oftalmol. 2019;82(3):239-41. 
47. Agaimy A, et al. Malignant teratoid tumor of the thyroid gland: an aggressive primitive multiphenotypic malignancy showing organotypical elements and frequent DICER1 alterations-is the term "thyroblastoma" more appropriate? Virchows Arch. 2020;477(6):787-98.

48. Choi S, et al. Dental abnormalities in individuals with pathogenic germline variation in DICER1. Am J Med Genet A. 2019;179(9):1820-5.

49. Schultz KAP, et al. Pleuropulmonary blastoma-like peritoneal sarcoma: a newly described malignancy associated with biallelic
DICER1 pathogenic variation. Mod Pathol. 2020;33(10):1922-9. https://pubmed.ncbi.nlm.nih.gov/32415267/.

Publisher's Note Springer Nature remains neutral with regard to jurisdictional claims in published maps and institutional affiliations. 